\title{
Flexible Control of Power Flow in Multiterminal DC Grids Using DC-DC Converter
}

\author{
Kumars Rouzbehi, Member, IEEE, Jose Ignacio Candela, Member, IEEE, Alvaro Luna, Member, IEEE, \\ Gevork B. Gharehpetian, Senior Member, IEEE, and Pedro Rodriguez, Fellow, IEEE
}

\begin{abstract}
This paper proposes an efficient control framework that utilizes dc-dc converters to achieve flexible power flow control in multiterminal dc (MTDC) grids. The dc-dc converter employed in this paper is connected in cascade with the dc transmission line, and is therefore named cascaded power flow controller (CPFC). In this paper, a two-layer control strategy is developed for the operation and control of voltage source converter stations and CPFC station in MTDC grids. At the primary control layer, a novel differential voltage droop control is developed, while at the secondary control layer, a modified de power flow algorithm-employing the new CPFC frameworkis implemented. The overall control strategy enables the CPFC to regulate the power flow in the de transmission line. The primary control guarantees the transient stability of the CPFC, and the secondary control system ensures the desired steady-state operation. The proposed voltage droop control framework helps the MTDC grid to remain stable in the event of a communication failure between the primary and secondary control layers. Static analysis and dynamic simulations are performed on the CIGRE B4 dc grid test system, in order to confirm the effectiveness of the proposed control framework for power flow regulation in MTDC grids.
\end{abstract}

Index Terms-CIGRE B4 de grid, de power flow control, multiterminal dc (MTDC) grid, power system control, voltage droop control.

\section{INTRODUCTION}

$\mathbf{I}$ $\mathrm{N}$ RECENT years, the multiterminal dc (MTDC) grid has emerged as a promising solution to meet future network requirements, with significant advantages and applications including the integration of offshore wind farms into mainland ac networks [1], the development of the so-called European offshore supergrid [2]-[4], and the interconnection of North Sea wind farms with Mediterranean solar plants and Scandinavian hydropower becoming a promising possibility [5].

In the near future, successful and reliable operation of MTDC grids will necessitate the development of flexible control infrastructures; this will force power systems and power

Manuscript received September 14, 2015; revised February 20, 2016 and April 13, 2016; accepted May 16, 2016. Date of publication June 7, 2016; date of current version July 29, 2016. Recommended for publication by Associate Editor Marta Molinas.

K. Rouzbehi, J. I. Candela, A. Luna, and P. Rodriguez are with the Department of Electrical Engineering, Technical University of Catalonia, Barcelona 08222, Spain (e-mail: kumars.rouzbehi@upc.edu; candela@ee.upc.edu; luna@ee.upc.edu; prodriguez@ee.upc.edu).

G. B. Gharehpetian is with the Department of Electrical Engineering, Amirkabir University of Technology, Tehran 15875-4413, Iran (e-mail: grptian@aut.ac.ir). electronics companies to develop appropriate control strategies in order to increase network reliability and efficiency [6]-[8].

At the primary control layer of an MTDC grid, the control of voltage source converter (VSC) stations is usually based on the vector current control technique, which provides fully decoupled control of ac grid active and reactive power quantities [8]. Voltage droop control is usually adopted for dc voltage control and power sharing within MTDC grids. Several papers have focused on the design and implementation of voltage-droopcontrol strategies for primary control of MTDC grids [8]-[13]. At the secondary control layer - the location of the supervisory control center-other issues such as dc load flow or optimal dc load flow should be addressed [11], [13].

Power flow control in MTDC grids is among the most important research topics addressed in recent years [14]-[16]. In MTDC grids, the flow of power through the dc lines depends solely on the voltage difference between both ends of the transmission line and the line resistance. At some operating points, there is risk of some lines becoming overloaded due to a lack of flexibility of the line to the power flow control. Another concern is loop power flows that can occupy more of the line's capacity, which has the potential to increase losses in the MTDC grid. These issues of line overloading and uncontrolled loop power flows, also known as the bottlenecks in the transmission lines, can be avoided with the use of power flow controllers.

Without having a power flow control device in the MTDC grid, there will not be enough capability of redirecting part of the power from one line to another line and delivering it to the objective ac area. It means that the construction of a new transmission line is necessary to avoid transmission line overload. Cost of adding a dc-dc converter to the existing transmission line is much lower than that of the construction of a new transmission line.

Due to the importance of the topic, CIGRE has initiated a working group, WG B4-58, to focus on the subject of load flow control devices in MTDC grids [17]. The working group is looking at the feasibility of power flow control in dc grids, to identify the methods and devices for controlling MTDC grid power flow. The utilization of dc-dc converters for power flow control in MTDC grids has already been proposed by CIGRE WG B4-58. However, this working group has not provided a specific control strategy for this power flow controller [17], [18]. In addition, aside from the need for a proper local control strategy for the dc-dc converters, in order to behave as dc power flow controllers, an appropriate 
global control strategy must be established to ensure the stable operation of MTDC grids in the event of contingencies.

The following problem is addressed by this paper in response to the abovementioned issues.

How to develop a complete control framework by employing a dc-dc converter to adjust the power flow on a specific dc transmission line to a predetermined reference value.

At the time of writing, there were several studies that focus on the use of dc-dc converters for power flow control in MTDC grids [15], [16], [19], [20], but they are centered on proposing power converter topologies, and have not addressed such converters' influence on the MTDC grid operation and control. There is also a lack of extant literature on the subject of the influence of dc-dc converter stations on the supervisory control of MTDC grids.

This paper contributes in this regard by proposing a complete control framework for the integration of power flow control into a dc-dc converter in an MTDC grid. Specifically, low- and high-level control structures for the $\mathrm{dc}-\mathrm{dc}$ converter are proposed that are integrated within the primary and secondary control layers. This paper also proposes a novel differential voltage droop controller for low-level control of the $\mathrm{dc}-\mathrm{dc}$ converter in an MTDC grid. Moreover, this paper integrates the proposed control structure of the power flow controller into the supervisory control center of the grid to regulate power flowing in a particular dc transmission line. Aside from being technically justified, such capability of a $\mathrm{dc}-\mathrm{dc}$ converter, can be highly beneficial from the market point of view, e.g., the shifting of power, transmitted between two zones, on to a particular line due to economic considerations as part of a transmission system congestion-management strategy [13], [21] or to provide improved flexibility as part of a loss-reduction strategy in an MTDC grid.

The study approach employed by this paper can be summarized as follows.

1) A dc-dc converter is used to enhance power flow controllability in an MTDC grid.

2) An average model is developed for the dc-dc converter that is suitable for static analysis; this is based on controlled current and voltage source. In order to make the model suitable for dynamic studies, an inductor and capacitor is integrated into this average model.

3) A local control structure is proposed for a dc-dc converter and its [cascaded power flow controller (CPFC)] stability is analyzed via a linearized small signal model.

4) A differential voltage droop scheme is proposed in the local control structure of the CPFC to account for grid transients.

5) The proposed local control structure of the CPFC is integrated into the supervisory control center of the MTDC grid to form a complete control framework.

This paper is organized as follows. Section II focuses on the flexibility of MTDC grid operation with the proposed local control structure in place for the CPFC. The integration of the CPFC into the dc power flow routine and, in general, in the control framework of the MTDC grid for the purposes of power flow control is presented in Section III. In order to demonstrate the capabilities of the $\mathrm{CPFC}$, static simulation

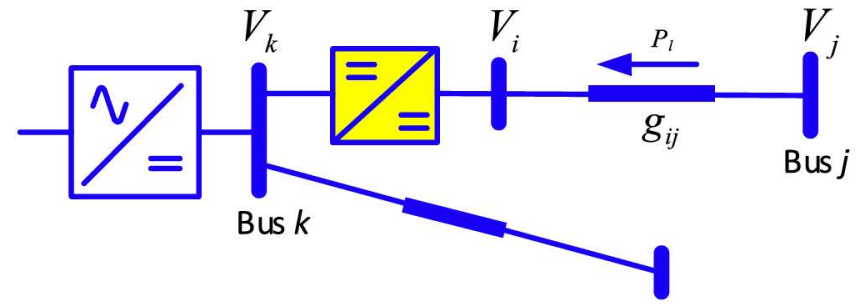

Fig. 1. Single-line representation of a dc-dc converter, connected to dc bus $k$.

results are presented in Section IV while dynamic evaluations based on CIGRE B4 MTDC grid test system are carried out in Section V. Finally, concluding remarks are summarized in Section VI.

\section{FleXible Operation of MTDC GRIDS}

Similar to FACTS devices in ac power systems, static devices are required to be developed and employed in MTDC grids in order to provide control over one or more dc transmission system parameters; enhancing grid controllability. Due to the advancements of power electronics technology, high-power dc-dc converters can provide power flow control functions in MTDC grids; enhancing the flexibility of grid operation [16]-[18], [23], [31]-[34]. By adjusting the dc-dc converter transformation ratio, the voltage at one terminal of the converter, or the power flowing through the converter, can be regulated, adding a degree of freedom to the control of the MTDC grid [22]. This is similar to the role of phase-shifting transformers in ac grids.

\section{A. Cascaded Power Flow Controller}

The CPFC [23], installed with cascade connection to the corresponding dc bus, shown in Fig. 1.

The CPFC will be able to control the power flowing through the dc line that is connected to bus $k$, by adjusting its transformation ratio $n_{c}$, defined as

$$
n_{c}=\frac{V_{k}}{V_{i}}
$$

where $V_{k}$ and $V_{i}$ indicate the steady-state de voltages on both sides of the CPFC in Fig. 1.

\section{B. Modeling of CPFC for Power System Studies}

As previously stated, the proposed CPFC is a dc-dc converter with a specific control structure that is devoted to the control of the power flow in a specific dc line. The CPFC must be appropriately modeled in order to be included in power system studies and analysis. The average model [21] of the CPFC, composed of controlled current and voltage sources, is illustrated in Fig. 2 [24].

In the average model of Fig. 2, the CPFC is modeled by a controlled current source $\left(I_{p}=n_{c} I_{k}\right)$ connected in parallel with a capacitor $\left(C_{p}\right)$ on the side connected to the dc line. On the other side, which is connected to the dc bus, the CPFC is represented by a voltage source $\left(V_{s}=n_{c} V_{i}\right)$ behind an inductor $\left(L_{S}\right)$. In this paper, this model is used to 


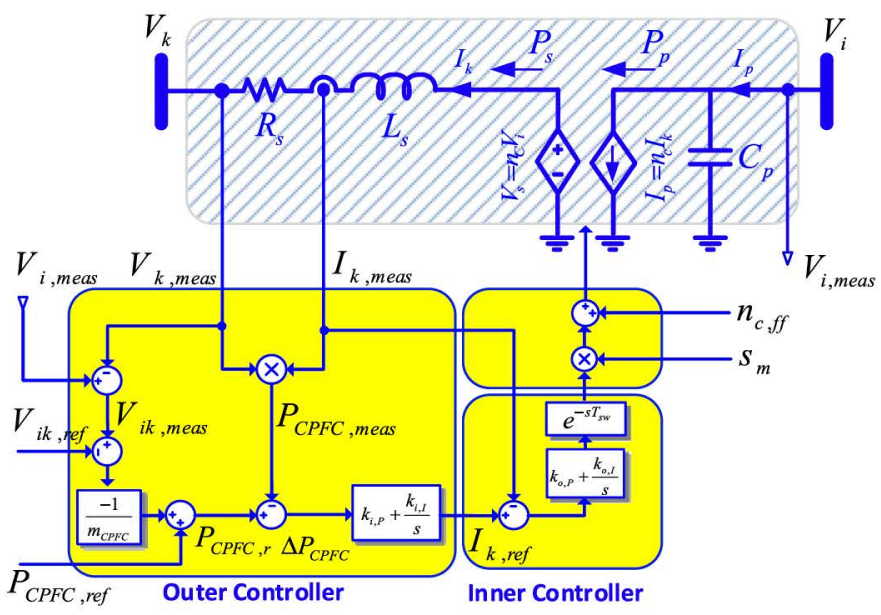

Fig. 2. Proposed control structure of the CPFC.

develop a two-port hybrid representation of the CPFC. This style of modeling is commonly applied to various power electronic converters [24]. For steady-state analyses and studies, the effect of the capacitor and the inductor vanish, and hence the following matrix representation, can be used:

$$
\left[\begin{array}{c}
V_{k} \\
I_{i}
\end{array}\right]=\left[\begin{array}{cc}
n_{C} & -R_{S} \\
0 & n_{C}
\end{array}\right]\left[\begin{array}{c}
V_{i} \\
I_{k}
\end{array}\right]
$$

The following frequency domain representation can be obtained for use with dynamic studies:

$$
\left[\begin{array}{l}
V_{k}(s) \\
I_{i}(s)
\end{array}\right]=\left[\begin{array}{cc}
n_{C} & -\left(R_{S}+s L_{S}\right) \\
s C_{P} & n_{C}
\end{array}\right]\left[\begin{array}{l}
V_{i}(s) \\
I_{k}(s)
\end{array}\right] .
$$

During steady-state conditions, (3) converges to (2).

\section{Local Control of the CPFC}

The local control system of the CPFC is shown in Fig. 2. The main objective of the CPFC local control is to achieve the desired power flow in the specific line with the use of an appropriate transformation ratio.

In the proposed control structure, two control modes are defined. The first mode, block mode, corresponds to a unity transformation ratio and hence does not provide any power flow regulation. The second mode is selected when power flow control is intended. Each mode is selected by a mode selection signal $s_{m}$ sent by the supervisory control system.

In addition, the reference power $P_{\mathrm{CPFC}, r}$ is provided by a differential voltage droop controller based on the measured voltage difference between both sides of the CPFC $V_{i k \text {,meas }}$ and the droop slope $m_{\mathrm{CPFC}}$. In the differential voltage droop strategy that is proposed, the voltage difference on both sides of the CPFC, i.e., $V_{i k}=V_{i}-V_{k}$, is used as the input of the voltage droop controller. The external loop of the CPFC also acts during transient states, in order to reduce the transient behavior and to change the local power reference.

It is worth noting that when the voltage difference is used, the CPFC can appear to the grid to be a virtual resistance. In fact, in this case, the power flowing through the CPFC changes according to the voltage difference on both sides; hence, the CPFC appears as a virtual resistance inside the grid. It must be noted that this type of voltage droop control differs from the voltage droop strategy employed for the control of VSC stations: for VSCs, the voltage on their dc side is used to calculate the reference power [25], [27].

The local control system in Fig. 2 comprises an inner and an outer loop. In the outer loop, the differential voltage droop scheme provides the CPFC reference current based on the difference between the reference and the measured powers $\left(\triangle P_{\mathrm{CPFC}}=P_{\mathrm{CPFC}, r}-P_{\mathrm{CPFC} \text {,meas }}\right)$. The inner control ( $\mathrm{IC}_{\mathrm{CPFC}}$ ) loop generates the control signal required to drive the measured current $I_{k \text {,meas }}$ to the reference value $I_{k \text {,ref }}$. The variable $n_{c, f f}$ is a feedforward transformation ratio that provides an initial value for $n_{c}$ in order to enhance the dynamic response of the CPFC local control system. The feedforward term is calculated from the outputs of the dc power flow program. This term is the steady-state value of the CPFC transformation ratio for the desired power of the controlled line. The addition of this term to the output of the CPFC control system improves the controller's performance.

To assess the stability of the CPFC, its closed-loop small signal model is linearized and illustrated in Fig. 3. In the linearized model, $m_{\mathrm{CPFC}}$ indicates the slope of the voltage droop characteristics. The CPFC delay due to the pulsewidth modulation (PWM) has been modeled using a second-order Padé approximation of the switching delay $e^{-s T_{\mathrm{sw}}}$ [denoted by Padé $\left(T_{\mathrm{sw}}, 2\right)$ in Fig. 3]. In the small signal model, the output of the inner PI controller is the appropriate transformation ratio.

The process of designing a robust controller requires a model that takes into consideration the uncertainties of the plant. In this paper, much research effort has been devoted to robustness for linear control systems with parametric uncertainties. The design of the controller for dc-dc and VSCs has followed by the well-known classical approach. The emphasis has been on stability margin computations for gain and phase of the feedback loop.

Considering several hundred mega-watts of power flow for our case study, the parameters of the PI controllers are tuned using linear control rules to obtain a settling time of $200 \mathrm{~ms}$ with a $2 \%$ overshoot.

The bode diagram of the open-loop system is shown in Fig. 4, and indicates the stable status of the open-loop system with a 26-dB gain margin and a phase margin of $77^{\circ}$. This diagram has been obtained using the internal parameters (i.e., the values of the resistor and inductor, and the delay due to PWM) of the dc-dc converter.

\section{INTEGRATION OF CPFC INTO THE CONTROL FRAMEWORK OF MTDC GRIDS}

At the primary control layer, the VSCs are locally controlled by the vector control in the $d q$ reference frame [6], [8]. The voltage-regulating VSCs adopt voltage droop control in the outer loop of their vector current control system to generate the appropriate reference currents for the inner current controllers in Fig. 5 [11], [28], [29]. The secondary control layer acts as a supervisory control system, sending appropriate control signals to the local controllers. At the secondary control layer, a power flow program is used to generate the appropriate signals for the local controllers, based on the current status in addition to the requirements of the MTDC grid. 


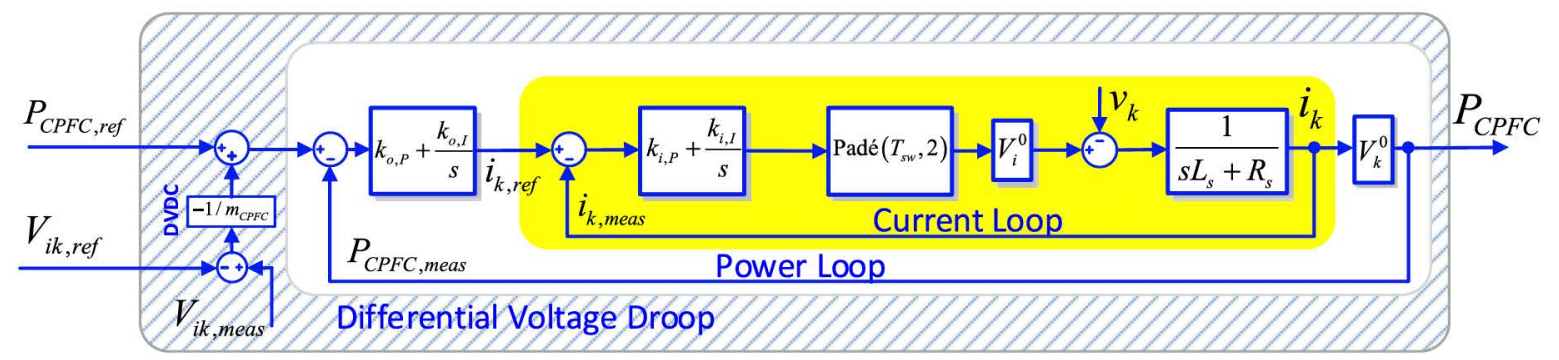

Fig. 3. Linearized small-signal model of the CPFC with power and current control loops.
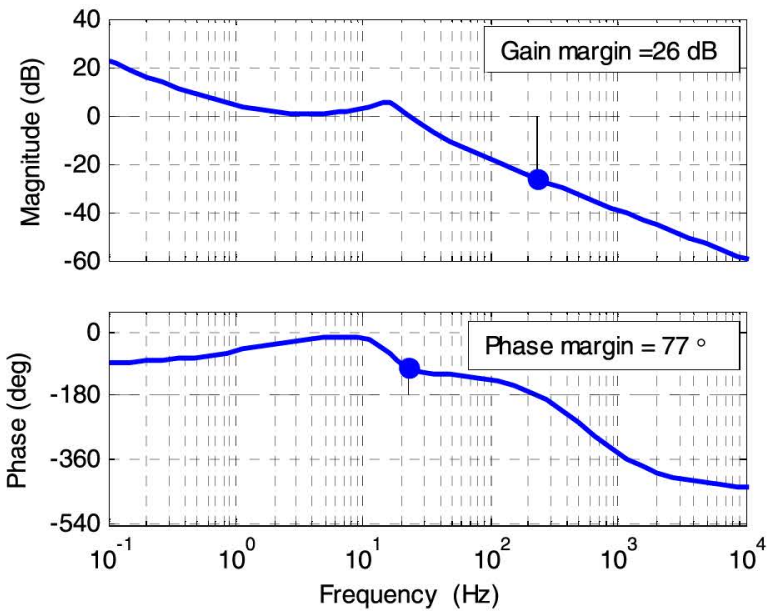

Fig. 4. Bode diagram of the CPFC open-loop small-signal model.

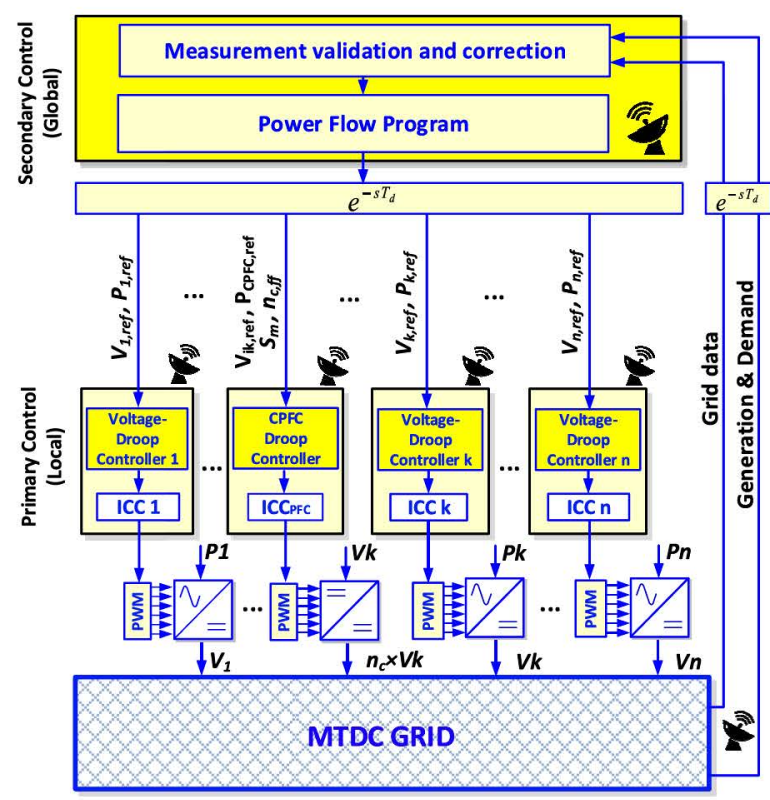

Fig. 5. Overall control structure for the MTDC grid with primary and secondary control layers.

This power flow program is executed at discrete intervals with the predefined secondary control sample time to provide settings for the primary controllers. In the proposed structure, as shown in Fig. 5, the communication delay between secondary and primary layers is also considered.

In order to capture the influence of the CPFC on the power flow in the dc lines, the CPFC must be included into the power flow program of the secondary control layer. To achieve this, it is necessary to reformulate the dc power flow routine.

\section{A. General Formulation of the MTDC Load Flow Problem}

Considering an $N$-terminal dc grid, the mathematical formulation of the dc load flow begins by enforcing the following power constraints on all dc buses:

$$
P_{i}=P_{G i}-P_{L i}=V_{i} I_{i}, i=1, \ldots, N
$$

where $P_{i}$ is the net power injected into dc bus $i$, computed as the difference between the power injected by generating elements $P_{G i}$ and the power absorbed by loads $P_{L i} ; V_{i}$ is the voltage of the dc bus $i$; and $I_{i}$ represents the net current injected into dc bus $i$ by the generators and the loads.

The net injected current of the bus $i I_{i}$ is related to the bus voltage $V_{i}$ through the conductance matrix of the MTDC grid

$$
\mathbf{I}=\mathbf{G V}
$$

where the vectors $\mathbf{I}=\left[\begin{array}{llll}I_{1} & I_{2} & \ldots & I_{N}\end{array}\right]$ and $\mathbf{V}=\left[\begin{array}{lll}V_{1} V_{2} & \ldots V_{N}\end{array}\right]^{T}$ contain the net injected currents and dc bus voltages of the MTDC grid, respectively, and $\mathbf{G}=\left[\mathcal{G}_{i j}\right]_{i, j=1, \ldots, N}$ represents the conductance matrix of the grid. The elements of $\mathbf{G}$ can be easily computed using the following equation:

$$
\mathcal{G}_{i i}=\sum_{j=1}^{N}\left(g_{i j}+g_{s i}\right), \quad \mathcal{G}_{i j}=-g_{i j}
$$

where $g_{i j}$ and $g_{s i}$ represent the conductance between dc buses $i$ and $j$, and the conductance between bus $i$ and ground, respectively. Finally, the power constraint on all grid buses in (4) can be restated as follows:

$$
P_{i}=V_{i} \sum_{j=1}^{N}\left(\mathcal{G}_{i j} V_{j}\right) \quad i=1, \ldots, N
$$

Based on these formulations, each dc bus adds two unknowns $P_{i}$ and $V_{i}$ to the MTDC grid load flow equations, resulting in a total of $2 \mathrm{~N}$ unknowns. There is a single equation associated with each dc bus, which means that we have $N$ equations. One variable per dc bus must, therefore, be specified in order to solve the load flow problem in (4). Depending on which variables are specified, two types of dc buses can be identified:

1) load (generation) or $P$-bus, whose net injected power is predefined $\left(P_{i}=P_{i}^{*}\right)$;

2) voltage or $V$-bus, whose dc voltage is predefined $\left(V_{i}=V_{i}^{*}\right)$. 
A slack bus, responsible for assuring power balance in the grid, must be considered in the dc power flow problem. As the dc voltage of the slack bus is predefined, it is eliminated from the load flow equations. Hence, the number of load flow equations reduces to $N-1$.

The Newton-Raphson (NR) method has been successfully applied to the problem of ac power system load flow [30]. Since the number of equations, as well as the constraints, for an ac load flow problem is higher than for MTDC load flow, it is expected that the NR method will produce a satisfactory solution to the load flow problem for MTDC grids.

By skipping the common mathematical details of the NR method, we can arrive at the solution of the MTDC load flow problem. Without any loss of generality, by assuming the first dc bus as the slack bus (and the only $V$-bus in the grid), the state variables can be expressed by

$$
\mathbf{V}=\left[V_{2} \ldots V_{N}\right]^{T}
$$

and the mismatch vector can be stated as

$$
\Delta \mathbf{P}=\left[\Delta P_{2} \Delta P_{3} \ldots \Delta P_{N}\right]^{T}
$$

where the elements of $\mathbf{\Delta P}$ are computed by the following equation:

$$
\Delta P_{i}=P_{i}^{*}-V_{i} \sum_{j=1}^{N} \mathcal{G}_{j} V_{j}, \quad i=2, \ldots, N .
$$

The updated state variables in the dc power flow problem at iteration $k$, i.e., $V_{2}, \ldots, V_{n}$, are obtained as follows:

$$
\begin{aligned}
{[\Delta \mathbf{V}]^{k+1} } & =[\mathbf{V}]^{k}+[\mathbf{\Delta} \mathbf{V}]^{k} \\
{[\Delta \mathbf{V}]^{k} } & =\left([\mathbf{J}]^{k}\right)^{-1} \cdot[\Delta \mathbf{P}]^{k}
\end{aligned}
$$

where $\mathbf{J}$ is the Jacobian matrix of the MTDC load flow, defined by the following equation:

$$
\mathbf{J}=\left[J_{i j}\right]_{i, j=2, \ldots, N}, \quad J_{i j}=-\partial P_{i} / \partial V_{j} .
$$

Based on (7), $J_{i j}$ can be expressed as follows:

$$
J_{i j}= \begin{cases}G_{i j} V_{i}, & i \neq j \\ -\sum_{\substack{j=1 \\ j \neq i}}^{N}\left(G_{i j} V_{j}\right)-2 G_{i j} V_{i}, & i=j\end{cases}
$$

\section{B. Inclusion of CPFC Into the MTDC Load Flow Problem}

The CPFC is designed to maintain an electrical quantity $y$ (i.e., line power flow) at a desired value $y^{*}$ by acting on the control variable $u$; it must therefore be incorporated into the dc power flow formulation. In this paper, this is done by the inclusion of the equality constraints imposed by the CPFC into the state and mismatch vectors of (12), so that

$$
\left[\begin{array}{c|c}
\mathbf{J} & -\partial \mathbf{P} / \partial \mathbf{u} \\
\hline-\partial \mathbf{y} / \partial \mathbf{V} & -\partial \mathbf{y} / \partial \mathbf{u}
\end{array}\right]^{k}\left[\frac{\Delta \mathbf{V}}{\Delta \mathbf{u}}\right]^{k}=\left[\frac{\boldsymbol{\Delta} \mathbf{P}}{\boldsymbol{\Delta} \mathbf{y}}\right]^{k}
$$

where

$$
\Delta \mathbf{y}=\mathbf{y}^{*}-\mathbf{y}
$$

are new elements of the mismatch vector and $\mathbf{u}$ represents the vector of the associated control variables.

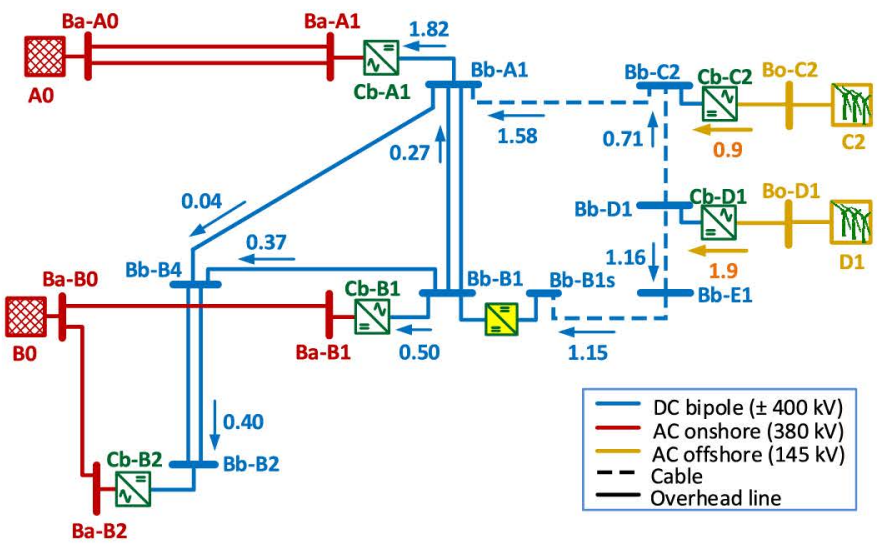

Fig. 6. Schematic diagram of DCS3 and detailed power flow results (rounded) for base case (CPFC losses are neglected and dc line powers are reported at the receiving ends).

In the case of an ideal CPFC (i.e., lossless CPFC), shown in Fig. 6, the following expressions can be derived:

$$
\begin{aligned}
P_{k j} & =P_{i j} \\
V_{k} & =n_{c} V_{i}
\end{aligned}
$$

where $P_{i j}$ is the power flowing from bus $i$ to bus $j$, and $n_{c}=V_{k} / V_{i}$ is the transformation ratio of the CPFC. By including the $\mathrm{CPFC}$, one degree of freedom is provided which allows for the regulation of either the dc voltage on one side of the converter or the power flowing through it. In both cases, $n_{c}$ is the only control variable

$$
\Delta u=\Delta n_{c} .
$$

When power regulation is intended, i.e., $P_{i j}=P_{i j}^{*}$, then we have

$$
\Delta y=\Delta P_{i j}=P_{i j}-P_{i j}^{*} .
$$

The Jacobian matrix can be updated to include the following elements, obtained from (18):

$$
\begin{aligned}
& \frac{\partial P_{i j}}{\partial V_{k}}=\frac{\partial P_{i j}}{\partial V_{i}} \cdot \frac{\partial V_{i}}{\partial V_{k}}=-\frac{1}{n_{c}} \cdot \frac{\partial P_{i j}}{\partial V_{i}} \\
& \frac{\partial P_{i j}}{\partial n_{c}}=\frac{\partial P_{i j}}{\partial V_{i}} \cdot \frac{\partial V_{i}}{\partial n_{c}}=-\frac{V_{k}}{n_{c}^{2}} \cdot \frac{\partial P_{i j}}{\partial V_{i}}
\end{aligned}
$$

where

$$
P_{i j}=V_{i}\left(V_{j}-V_{i}\right)\left(-\mathcal{G}_{i j}\right) .
$$

Hence, (15) becomes

$$
\left[\begin{array}{c|c}
\mathbf{J} & -\partial \mathbf{P} / \partial n_{c} \\
\hline-\partial P_{i j} / \partial \mathbf{V} & -\partial P_{i j} / \partial n_{c}
\end{array}\right]^{k}\left[\frac{\Delta \mathbf{V}}{\Delta n_{c}}\right]^{k}=\left[\frac{\Delta \mathbf{P}}{\Delta P_{i j}}\right]^{k}
$$

and

$$
\begin{aligned}
\partial P_{i j} / \partial V_{k} & =\frac{1}{n_{c}}\left(V_{j}-2 V_{i}\right)\left(-\mathcal{G}_{i j}\right) \\
& =\frac{1}{n_{c}}\left(V_{j}-2 \frac{V_{k}}{n_{c}}\right)\left(-\mathcal{G}_{i j}\right) \\
\partial P_{i j} / \partial V_{j} & =V_{i}\left(-\mathcal{G}_{i j}\right) \\
& =\frac{V_{k}}{n_{c}}\left(-\mathcal{G}_{i j}\right) \\
\partial P_{i j} / \partial n_{c} & =-\frac{V_{k}}{n_{c}^{2}}\left(V_{j}-2 V_{i}\right)\left(-\mathcal{G}_{i j}\right) \\
& =-\frac{V_{k}}{n_{c}^{2}}\left(V_{j}-2 \frac{V_{k}}{n_{c}}\right)\left(-\mathcal{G}_{i j}\right) .
\end{aligned}
$$


TABLE I

OverheAd Line AND CABLE DATA

\begin{tabular}{ccccc}
\hline \hline Line Data & $\begin{array}{c}\mathbf{R} \\
{[\boldsymbol{\Omega} / \mathbf{k m}]}\end{array}$ & $\begin{array}{c}\mathbf{L} \\
{[\mathbf{m H} / \mathbf{k m}]}\end{array}$ & $\begin{array}{c}\mathbf{C} \\
{[\boldsymbol{\mu F} / \mathbf{k m}]}\end{array}$ & $\begin{array}{c}\mathbf{G} \\
{[\boldsymbol{\mu S} / \mathbf{k m}]}\end{array}$ \\
\hline DC OHL $\pm \mathbf{4 0 0 k V}$ & 0.0114 & 0.9356 & 0.0123 & - \\
DC Cable $\pm \mathbf{4 0 0 k V}$ & 0.0095 & 2.1120 & 0.1906 & 0.048 \\
AC OHL 380kV & 0.0200 & 0.8532 & 0.0135 & - \\
\hline \hline
\end{tabular}

TABLE II

POWER FLOW DATA

\begin{tabular}{lccc}
\hline \hline DC Bus & Bus Type & DC Voltage & Net Power (pu) \\
\hline Bb-A1 & Slack & 1 & Unknown \\
Bb-B1 & $P$ & Unknown & -0.5 \\
Bb-B1s & Intermediate & Unknown & 0 \\
Bb-B2 & $P$ & Unknown & -0.4 \\
Bb-B4 & Intermediate & Unknown & 0 \\
Bb-C2 & $P$ & Unknown & 0.9 \\
Bb-D1 & $P$ & Unknown & 1.9 \\
Bb-E1 & Intermediate & Unknown & 0 \\
\hline \hline
\end{tabular}

The augmented Jacobian matrix in (24) and expressions in (25)-(27) allow inclusion of the CPFC into the dc power flow program. In (24), the additional row and column are associated with the inclusion of the CPFC, and introduce the transformation ratio of the CPFC $n_{c}$ as a new power flow unknown.

\section{Static Simulation Results}

\section{A. Test MTDC Grid}

In order to demonstrate how the CPFC capabilities enhance the controllability of the MTDC grid, static simulations are carried out on the CIGRE B4 MTDC grid test system [18]. This test system is developed by CIGRE's B4 working group as a benchmark for conducting MTDC grid studies [17]. The CIGRE B4 MTDC grid test system includes two onshore ac systems, four offshore ac systems, two dc buses (with no connection to any ac system), and three MTDC systems.

In this paper, the MTDC system three (DCS3), a five-terminal meshed grid comprised of bipolar VSC-HVdc stations with dc-link voltage of $\pm 400 \mathrm{kV}$, is employed in order to evaluate the proposed control framework. The schematic diagram of DCS3 is shown in Fig. 6.

The data for the dc transmission system are presented in Table I. Note that the base power and voltage are $500 \mathrm{MW}$ and $800 \mathrm{kV}$, respectively.

As can be seen in Fig. 6, a dc-dc converter is installed between dc buses Bb-B1 and Bb-B1s. This converter plays the role of the dc power flow controller in DCS3 and provides special control over the power flow in the dc line between $\mathrm{dc}$ buses $\mathrm{Bb}-\mathrm{E} 1$ and $\mathrm{Bb}-\mathrm{B} 1$. It is worth noting that without the presence of the CPFC, the VSC installed at the dc bus Bb-B1 (i.e., $\mathrm{Cb}-\mathrm{B} 1$ ) will not be able to simultaneously control the power of the $\mathrm{dc}$ bus and the dc line between the dc buses $\mathrm{Bb}-\mathrm{B} 1$ and Bb-E1.

The power flow data, i.e., slack and P-bus assumptions, are presented in Table II where Bb-A1 is the grid slack bus,
TABLE III

Power Flow Results (Base CASe: For $n_{c}=1$ )

\begin{tabular}{cccc}
\hline \hline DC Bus/DC Line & Bus Type & DC Voltage (pu) & Net Power (pu) \\
\hline Bb-A1 & Slack & 1 & -1.8254 \\
Bb-B1 & P & 1.001 & -0.5000 \\
Bb-B1s & Intermediate & 1.001 & 0 \\
Bb-B2 & P & 0.9986 & -0.4000 \\
Bb-B4 & Intermediate & 0.9996 & 0 \\
Bb-C2 & $\mathrm{P}$ & 1.0047 & 0.9000 \\
Bb-D1 & $\mathrm{P}$ & 1.0079 & 1.9000 \\
Bb-E1 & Intermediate & 1.0044 & 0 \\
Bb-E1 to Bb-B1s $^{*}$ & - & - & 1.1515 \\
Bb-C2 to Bb-A1 & - & - & 1.5873 \\
\hline \hline
\end{tabular}

"Line powers are reported at the receiving end.

and $\mathrm{Bb}-\mathrm{B} 1$ and $\mathrm{Bb}-\mathrm{B} 2$ are $P$-buses with predefined net injected power.

Two offshore buses, i.e., $\mathrm{Bb}-\mathrm{C} 2$ and $\mathrm{Bb}$-D1, are also $P$-bus and have predefined generation. Note that in the diagram of Fig. 6, dc buses Bb-B4 and Bb-B1s are intermediate dc buses with no load and no generation.

\section{B. Base Case: No Power Flow Control by CPFC}

In the base case, the transformation ratio of the CPFC is kept at unity $\left(n_{c}=1\right)$, and hence, the CPFC does not provide power flow control. By applying the NR method to the current dc power flow problem, the dc voltage and net injected power of all buses in DCS3 are obtained, as presented in Table III. More detailed results are shown in Fig. 6.

\section{Case 1: Line Power Control by CPFC}

In this case, the CPFC is used to control the power transmitted through the dc line between $\mathrm{Bb}-\mathrm{B} 1$ and $\mathrm{Bb}-\mathrm{E} 1$. Specifically, the power flowing from Bb-E1 to Bb-B1 should be maintained at $1.4 \mathrm{pu}$ (note that the power flowing through this line was $1.15 \mathrm{pu}$ in the base case), with all other power flow assumptions (i.e., generation and consumption in the DCS3 buses) being satisfied.

The power flow problem is formulated and solved using (15)-(24). Based on the resulting power flow solution, in order to achieve the desired power flow through the dc line between $\mathrm{Bb}-\mathrm{B} 1$ and $\mathrm{Bb}-\mathrm{E} 1$, the $\mathrm{CPFC}$ transformation ratio must be set to $n_{c}=1.0041$. The results of the power flow for this case are summarized in Table IV.

Note that while the power flowing from $\mathrm{Bb}-\mathrm{E} 1$ to $\mathrm{Bb}-\mathrm{B} 1$ has been adjusted to $1.4 \mathrm{pu}$, the net injected power in the $\mathrm{dc}$ buses is the same as the base case, with the exception of the slack bus. When the power flow through a specific line is changed, it is highly likely that this will lead to a change in the total system losses, which must then be compensated for by the slack bus, i.e., the injected power in the slack bus should change. A comparison of the results presented in Tables III and IV indicates that the dc voltage of all buses in case 1 has changed with respect to the base case; this is due to the voltage regulation provided by the CPFC. The detailed 
TABLE IV

POWER Flow RESUlts (CASE 1: FOR $n_{c}=1.0041$ )

\begin{tabular}{|c|c|c|c|}
\hline DC Bus/DC Line & Bus Type & DC Voltage (pu) & Net Power (pu) \\
\hline Bb-A1 & Slack & 1 & -1.8254 \\
\hline Bb-B1 & $\mathrm{P}$ & 1.0017 & -0.5000 \\
\hline Bb-B1s & Intermediate & 0.9976 & 0 \\
\hline Bb-B2 & $\mathrm{P}$ & 0.9991 & -0.4000 \\
\hline Bb-B4 & Intermediate & 1.0002 & 0 \\
\hline $\mathrm{Bb}-\mathrm{C} 2$ & $\mathrm{P}$ & 1.0040 & 0.9000 \\
\hline Bb-D1 & $\mathrm{P}$ & 1.0060 & 1.9000 \\
\hline Bb-E1 & Intermediate & 1.0018 & 0 \\
\hline Bb-E1 to Bb-B1s* & - & - & 1.4000 \\
\hline $\mathrm{Bb}-\mathrm{C} 2$ to $\mathrm{Bb}-\mathrm{A} 1^{*}$ & - & - & 1.3386 \\
\hline
\end{tabular}

* Line powers are reported at the receiving end.

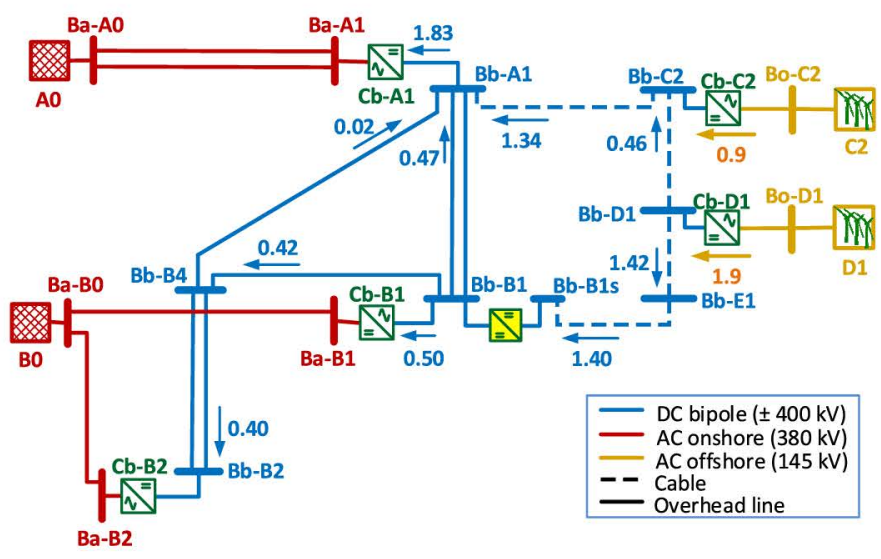

Fig. 7. Detailed power flow results (rounded) for case 1 (CPFC losses are neglected and dc line powers are reported at receiving ends).

power flow results for case 1 are illustrated in Fig. 7, where the power flowing through all dc lines is presented.

Note that in Fig. 7, the sum of incoming and outgoing power at every node may deviate from zero; this is due to the omission of de transmission losses. The change in power flow on all of the dc lines, with respect to the base case, can be observed by comparing Fig. 6 with Fig. 7 .

\section{Dynamic PERformance Evaluation}

This section of this paper presents the results of the dynamic simulations that were carried out on DCS3, employing the average model of the VSC and CPFC stations.

In DCS3, the grid-side VSCs Cb-A1, Cb-B1, and Cb-B2 all adopt voltage droop characteristics and hence contribute to the dc voltage control and power sharing in the MTDC grid. Contrastingly, the wind farm-side VSCs $\mathrm{Cb}-\mathrm{C} 2$ and $\mathrm{Cb}-\mathrm{D} 1$, are used to control the frequency of their corresponding ac grids.

\section{A. Dynamic Evaluation of Power Flow Control by the CPFC}

In this simulation, the control mode of the CPFC is set to power control (i.e., control mode 2 in Fig. 2). The simulation is initiated using the power flow results for the static base case, summarized in Table III. The parameters of the voltage droop

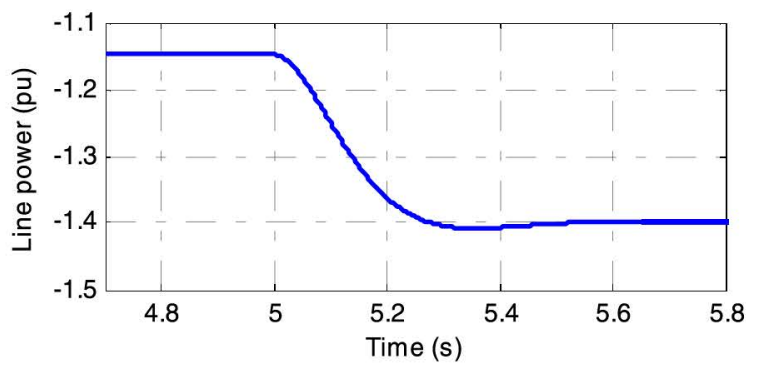

Fig. 8. Controlled power of the line between $\mathrm{Bb}-\mathrm{B} 1$ and $\mathrm{Bb}-\mathrm{E} 1$ for $P_{\mathrm{CPFC}, \text { ref }}=1.4 \mathrm{pu}$.

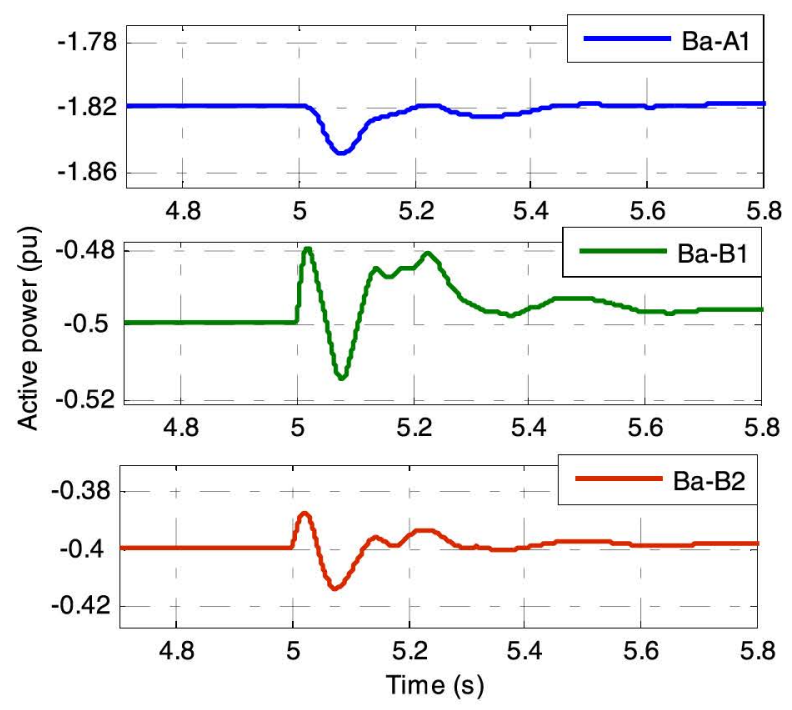

Fig. 9. Active power of the grid-side VCSs for PCPFC, ref $=1.4 \mathrm{pu}$.

characteristic of the grid-side VSCs are tuned according to the dc power flow results.

In this simulation, prior to $t=5 \mathrm{~s}, S_{m}=0$, and $n_{c, f f}=1$; i.e., no control action is provided by the CPFC.

At this stage of the simulation, the steady-state active powers and dc voltages are in line with the base case power flow results, presented in Table III. The line power flow between $\mathrm{Bb}-\mathrm{B} 1$ and $\mathrm{Bb}-\mathrm{E} 1$ and the active power at the grid-side VSCs are shown in Figs. 8 and 9, respectively.

At $t=5 \mathrm{~s}$, the supervisory control system executes a new dc power flow to adjust the power transmitted by the line between $\mathrm{Bb}-\mathrm{E} 1$ and $\mathrm{Bb}-\mathrm{B} 1$ at $1.4 \mathrm{pu}$ voltage. Based on the results presented in Table IV, the transformation ratio of the CPFC must be adjusted to $n_{c}=1.0041$. The CPFC voltage droop parameters, the feedforward control signal $\left(n_{c}=1.0041\right)$, and the control mode selection signal $\left(s_{m}=1\right)$ are sent by the secondary control layer to the local controller of the CPFC. The voltage droop characteristics of the grid-side VSCs are retuned based on the results of the new dc power flow. It can be seen from Fig. 8 that the power flow through the controlled line has reached the new reference value, with a settling time of $200 \mathrm{~ms}$, thus demonstrating the desired performance of the control system. It is worth noting that this settling time is directly related to the parameters of the CPFC controller as well as to the inductance and resistance. Note that the size 


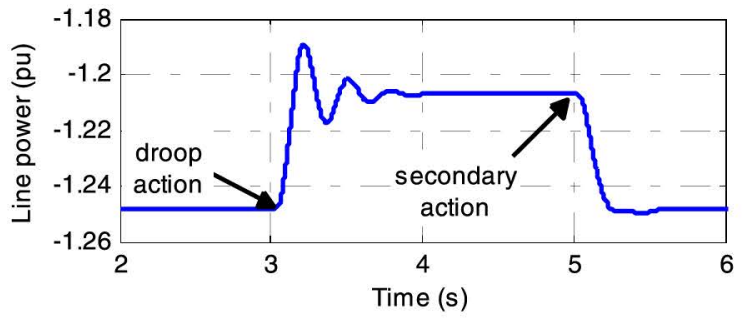

Fig. 10. Controlled power of the line between Bb-B1 and Bb-E1 VCSs during grid disturbance.
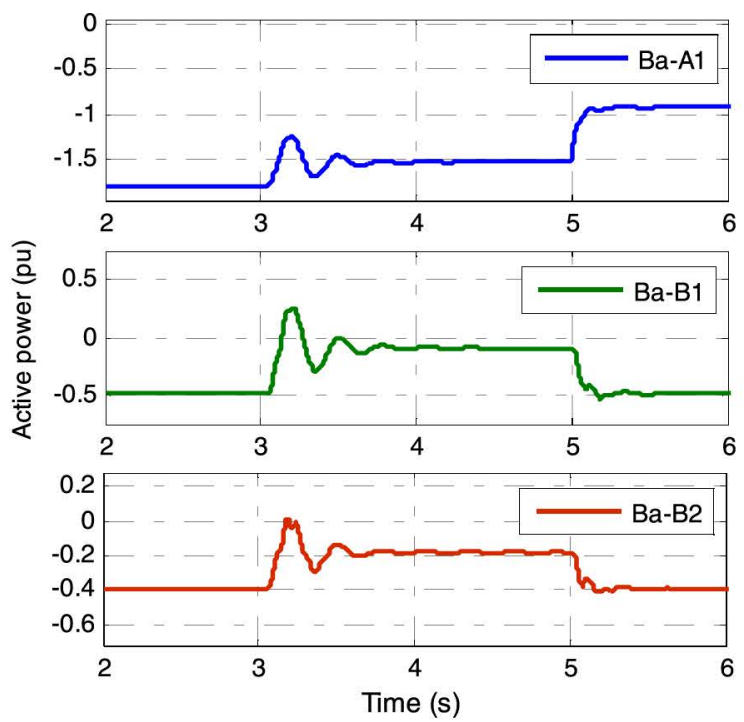

Fig. 11. Active power of the grid-side VCSs during grid disturbance.

of $L_{S}$ and $R_{S}$ is very small in comparison with the inductance and resistance of the cables.

Based on the active power of the grid-side VSCs shown in Fig. 9, the droop-based voltage control system of the VSCs has successfully maintained the active power of the VSC stations at their predefined value. This indicates the satisfactory performance of both the local and the global control systems.

\section{B. Dynamic Evaluation in Case of Disturbances}

The capability of the CPFC to control the power of a specific dc line through its regulatory action has been demonstrated. It is, however, important to verify how the CPFC performs when a disturbance (in generation or on the demand side) occurs in the MTDC grid. In this scenario, the power transmitted through the line between Bb-B1 and Bb-E1 is initially controlled at $1.25 \mathrm{pu}$ by the CPFC. Then, the generation of the offshore grid Bo-D1 is reduced from 1.9 to $1 \mathrm{pu}$ at $t=3 \mathrm{~s}$. This large disturbance changes the status of the grid, and it is crucial that during this event, the CPFC does not lead to the instability of the MTDC grid.

Fig. 10 shows the power flow through the controlled line during this simulation. It is seen that when the disturbance occurs, the power - controlled by the CPFC - is reduced due to its voltage droop control action. As the total generated power is significantly reduced, the power transmitted by the controlled

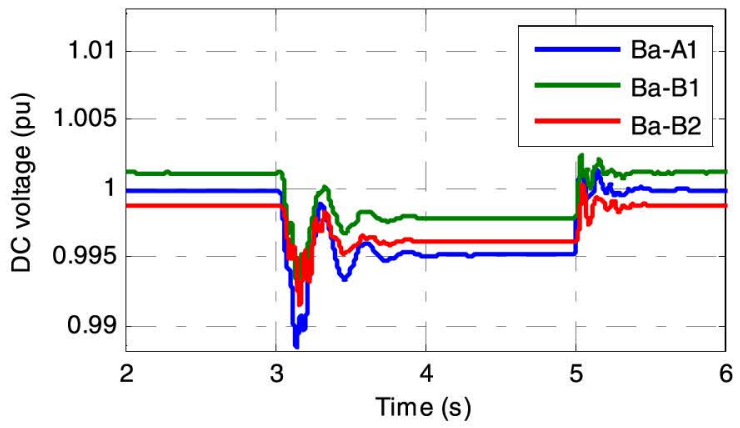

Fig. 12. DC voltage of the grid-side VCSs during grid disturbance.

line decreases, as a result of the voltage droop control action of the CPFC. At $t=5 \mathrm{~s}$, new set points for the voltage droop controllers of the grid-side VSCs, and the CPFC, are calculated and sent by the secondary controller. This is done by executing a new power flow calculation for the new grid status, and the demand requirements of $\mathrm{Ba}-\mathrm{B} 1$ and $\mathrm{Ba}-\mathrm{B} 2$. As can be seen from Fig. 10, the power flow of the controlled line is restored back to 1.25 pu. Fig. 11 shows that the grid-side VSCs $\mathrm{Cb}-\mathrm{B} 1$ and $\mathrm{Cb}-\mathrm{B} 2$ each receive their predefined power, ( 0.5 and $0.4 \mathrm{pu}$, respectively), after the retuning of the voltage droop controllers by the secondary control. The dc voltage profile of the grid-side VSCs during this simulation is depicted in Fig. 12.

\section{CONCLUSION}

This paper proposed a CPFC to regulate the power flowing through a dc transmission line within an MTDC grid. A complete two-layer control framework for VSC and CPFC stations was proposed and analyzed. At the primary control layer, a novel voltage-droop-control strategy was employed while, at the secondary control layer, the dc power flow algorithm was modified to take into account the effects of the power flow controller. Through the application of the proposed control strategy, the CPFC can reschedule the power flow in an MTDC grid to increase the utilization of dc lines, while improving the grid's efficiency and avoiding bottlenecks. The flexibility that this control strategy provides for the MTDC grid was shown through sound mathematical analysis as well as static and dynamic simulations on the CIGRE B4 dc grid test system.

\section{REFERENCES}

[1] L. Jun, J. Tianjun, O. Gomis-Bellmunt, J. Ekanayake, and N. Jenkins, "Operation and control of multiterminal HVDC transmission for offshore wind farms," IEEE Trans. Power Del., vol. 26, no. 4, pp. 2596-2604, Oct. 2011.

[2] U. N. A. Chowdhury and A. Yanushkevich, "Power flow analysis of meshed AC-DC super grid," in Proc. IEEE Eindhoven PowerTech, Jun./Jul. 2015, pp. 1-6.

[3] D. Van Hertem and M. Ghandhari, "Multi-terminal VSC HVDC for the European supergrid: Obstacles," Renew. Sustain. Energy Rev., vol. 14, no. 9, pp. 3156-3163, Dec. 2010.

[4] L. Xu, B. W. Williams, and L. Yao, "Multi-terminal DC transmission systems for connecting large offshore wind farms," in Proc. IEEE PES General Meeting, Jul. 2008, pp. 1-7.

[5] J. Blau, "Europe plans a North Sea grid," IEEE Spectr, vol. 47, no. 3, pp. 12-13, Mar. 2010.

[6] N. R. Chaudhuri and B. Chaudhuri, "Adaptive droop control for effective power sharing in multi-terminal DC (MTDC) Grids," IEEE Trans. Power Syst., vol. 28, no. 1, pp. 21-29, Feb. 2013. 
[7] A. S. Abdel-Khalik, A. M. Massoud, A. A. Elserougi, and S. Ahmed, "Optimum power transmission-based droop control design for multiterminal HVDC of offshore wind farms," IEEE Trans. Power Syst., vol. 28, no. 3, pp. 3401-3409, Aug. 2013.

[8] K. Rouzbehi, A. Miranian, J. I. Candela, A. Luna, and P. Rodriguez, "A generalized voltage droop strategy for control of multiterminal DC grids," IEEE Trans. Ind. Appl., vol. 51, no. 1, pp. 607-618, Jan./Feb. 2015.

[9] T. K. Vrana, J. Beerten, R. Belmans, and O. B. Fosso, "A classification of DC node voltage control methods for HVDC grids," Electr. Power Syst. Res., vol. 103, pp. 137-144, Oct. 2013.

[10] J. Beerten, S. Cole, and R. Belmans, "Modeling of multi-terminal VSC HVDC systems with distributed DC voltage control," IEEE Trans. Power Syst., vol. 29, no. 1, pp. 34-42, Jan. 2014.

[11] K. Rouzbehi, A. Miranian, J. I. Candela, A. Luna, and P. Rodriguez, "A hierarchical control structure for multi-terminal VSC-based HVDC grids with GVD characteristics," in Proc. ICRERA, Oct. 2013, pp. 996-1001.

[12] T. K. Vrana, L. Zeni, and O. B. Fosso, "Active power control with undead-band voltage \& frequency droop applied to a meshed DC grid test system," in Proc. ENERGYCON, Sep. 2012, pp. 612-616.

[13] K. Rouzbehi, A. Miranian, A. Luna, and P. Rodriguez, "DC voltage control and power sharing in multiterminal DC grids based on optimal DC power flow and voltage-droop strategy," IEEE J. Emerg. Sel. Topics Power Electron., vol. 2, no. 4, pp. 1171-1180, Dec. 2014.

[14] W. Wang and M. Barnes, "Power flow algorithms for multi-terminal VSC-HVDC with droop control," IEEE Trans. Power Syst., vol. 29, no. 4, pp. 1721-1730, Jul. 2014.

[15] E. Veilleux and B.-T. Ooi, "Multiterminal HVDC with thyristor powerflow controller," IEEE Trans. Power Del., vol. 27, no. 3, pp. 1205-1212, Jul. 2012.

[16] D. Jovcic, M. Hajian, H. Zhang, and G. Asplund, "Power flow control in DC transmission grids using mechanical and semiconductor based DC/DC devices," in Proc. IEEE ACDC, Dec. 2012, pp. 1-6.

[17] CIGRE WG B4-58: Load Flow Control and Direct Voltage Control in a Meshed HVDC Grid, accessed on May 9, 2016. [Online]. Available: http://b4.cigre.org

[18] CIGRE B4 Working Group, accessed on May 9, 2016. [Online]. Available: http://b4.cigre.org/publications

[19] Q. Mu, J. Liang, Y. Li, and X. Zhou, "Power flow control devices in DC grids," in Proc. IEEE PES General Meeting, San Diego, CA, USA, Jul. 2012, pp. 1-7.

[20] Y. Zhou, D. E. Macpherson, W. Blewitt, and D. Jovcic, "Comparison of DC-DC converter topologies for offshore wind-farm application," in Proc. PEMD, Mar. 2012, pp. 1-6.

[21] B. Chong, X. P. Zhang, L. Yao, K. R. Godfrey, and M. Bazargan, "Congestion management of electricity markets using FACTS controllers," in Proc. IEEE PES General Meeting, Jun. 2007, pp. 1-6.

[22] N. M. Peterson and W. S. Meyer, "Automatic adjustment of transformer and phase-shifter taps in the Newton power flow," IEEE Trans. Power App. Syst., vol. PAS-90, no. 1, pp. 103-108, Jan. 1971.

[23] D. Jovcic, "Bidirectional, high-power DC transformer," IEEE Trans. Power Del., vol. 24, no. 4, pp. 2276-2283, Oct. 2009.

[24] R. W. Erickson and D. Maksimovic, Fundamentals of Power Electronics, 2nd ed. NJ, USA: Springer, 2001.

[25] T. M. Haileselassie and K. Uhlen, "Precise control of power flow in multiterminal VSC-HVDCs using DC voltage droop control," in Proc. IEEE PES General Meeting, Jul. 2012, pp. 1-9.

[26] A. Egea-Álvarez, F. Bianchi, O. Gomis-Bellmunt, A. Junyent-Ferre, and G. Gross, "Voltage control of multiterminal VSC-HVDC transmission systems for offshore wind power plants: Design and implementation in a scaled platform," IEEE Trans. Ind. Electron., vol. 60, no. 6, pp. 2381-2391, Jun. 2013.

[27] J. Beerten and R. Belmans, "Analysis of power sharing and voltage deviations in droop-controlled DC grids," IEEE Trans. Power Syst. vol. 28, no. 4, pp. 4588-4597, Nov. 2013.

[28] M. Aragüés-Peñalba, A. Egea-Álvarez, O. Gomis-Bellmunt, and A. Sumper, "Optimum voltage control for loss minimization in HVDC multi-terminal transmission systems for large offshore wind farms," Electr. Power Syst. Res., vol. 89, pp. 54-63, Aug. 2012.

[29] K. Rouzbehi, C. Gavriluta, J. I. Candela, A. Luna, and P. Rodriguez, "Comprehensive analogy between conventional AC grids and DC grids characteristics," in Proc. IECON, Nov. 2013, pp. 2004-2010.

[30] A. Gomez-Exposito, A. J. Conejo, and C. Canizares, Eds., Electric Energy Systems: Analysis and Operation. New York, NY, USA: Taylor \& Francis, 2009
[31] S. P. Engel, N. Soltau, H. Stagge, and R. W. De Doncker, "Improved instantaneous current control for high-power three-phase dual-active bridge DC-DC converters," IEEE Trans. Power. Electron., vol. 29, no. 8, pp. 4067-4077, Aug. 2014.

[32] S. P. Engel, M. Stieneker, N. Soltau, S. Rabiee, H. Stagge, and R. W. De Doncker, "Comparison of the modular multilevel DC converter and the dual-active bridge converter for power conversion in HVDC and MVDC grids," IEEE Trans. Power Electron., vol. 30, no. 1, pp. 124-137, Jan. 2015.

[33] D. Jovcic and L. Zhang, "LCL DC/DC converter for DC grids," IEEE Trans. Power Del., vol. 28, no. 4, pp. 2071-2079, Oct. 2013.

[34] Y. Hu, R. Zeng, W. Cao, J. Zhang, and S. J. Finney, "Design of a modular, high step-up ratio DC-DC converter for HVDC applications integrating offshore wind power,' IEEE Trans. Ind. Electron., vol. 63, no. 4, pp. 2190-2202, Apr. 2016, doi: 10.1109/TIE.2015.2510975.

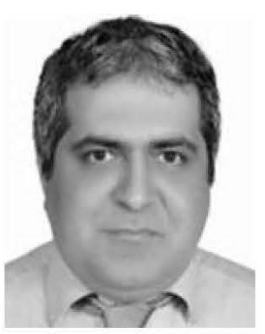

Kumars Rouzbehi (S'13-M'16) received the B.E.E and M.E.E. (Hons.) degrees in electrical engineering from IAU in 1998 and 2001, respectively, and the Ph.D. (cum laude) degree in electric energy systems from the the Technical University of Catalonia (UPC), Barcelona, Spain, in 2016.

He joined the Faculty of Electrical Engineering, IAU, as a Faculty Member in 2004, where he became the Director of the Department of Electrical Engineering. In parallel with teaching and research at IAU, he was the CEO of Khorasan Electric and Electronics Industries Researches Company from 2004 to 2010. He holds a patent in AC grid synchronization of voltage source converters and has coauthored several technical books, ISI journal papers, and technical conference proceedings.

Dr. Rouzbehi has been a member of the Amvaje-e-bartar (an Iranian journal of electrical engineering) Policy Making Committee and has served as an Associate Editor since 2006. He was a recipient of the Second Best Paper Award 2015 from the IEEE Power Electronic Society and the IEEE JOURNAL of Emerging and Selected Topics on Power Electronics. He has been a TPC Member of the International Conference on Electronic, Communication Control and Power Engineering (IEEE-ECCP) since 2014 and a Scientific Board Member of the (IEA) International Conference on Technology And Energy Management since 2015.

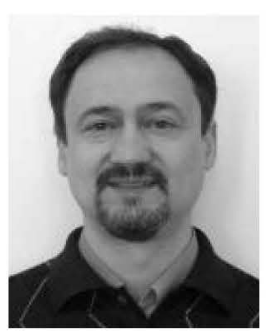

Jose Ignacio Candela (S'99-M'04) received the B.S., M.S., and Ph.D. degrees from the Technical University of Catalonia (UPC), Terrassa, Spain, in 1987, 2000, and 2009, respectively, all in industrial engineering.

He became an Assistant Professor in 1991 and has been an Associate Professor since 1993 with UPC. He has authored or co-authored more than 30 published technical papers and has been involved in several industrial projects and educational programs in the fields of power quality conditioning and motor drives. His current research interests include power conditioning, integration of distributed energy systems, and control of power converters and motor drives.

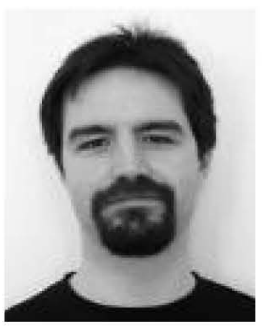

Alvaro Luna (S'07-M'10) received the B.Sc., M.Sc., and Ph.D. degrees from the Technical University of Catalonia (UPC), Barcelona, Spain, in 2001, 2005, and 2009, respectively, all in electrical engineering.

He joined UPC in 2005, as a Faculty Member, where he is currently an Assistant Professor. His current research interests include wind turbines control, PV systems, integration of distributed generation, and power conditioning. 


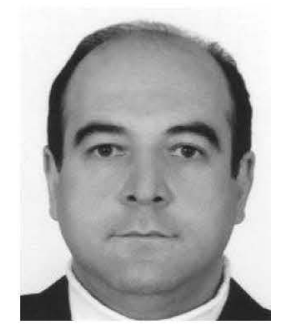

Gevork B. Gharehpetian (M'00-SM'08) received the B.S. (Hons.) degree from Tabriz University, Tabriz, Iran, in 1987, the M.S. (Hons.) degree from the Amirkabir University of Technology (AUT), Tehran, Iran, in 1989, and the Ph.D. (Hons.) degree from Tehran University, Tehran, in 1996, all in electrical engineering.

He was with the High Voltage Institute, RWTH Aachen, Aachen, Germany. He was an Assistant Professor with AUT from 1997 to 2003, where he was the Associate Professor from 2004 to 2007 and has been a Professor since 2007. He was selected by the Ministry of Higher Education as the Distinguished Professor of Iran and by the Iranian Association of Electrical and Electronics Engineers (IAEEE) as the Distinguished Researcher of Iran. He has authored over 900 journal and conference papers. His current research interests include smart grid, microgrids, FACTS and HVdc systems, and monitoring of power transformers and their transients.

Prof. Gharehpetian is a Distinguished Member of IAEEE and a member of the Central Board of IAEEE. As a Ph.D. student, he received scholarship from DAAD (German Academic Exchange Service) from 1993 to 1996. He received the National Prize in 2008 and 2010. Based on the ISI Web of Science database from 2005 to 2015, he is among the world's top 1\% of elite Scientists, according to Essential Science Indicators' ranking system. Since 2004, he has been the Editor-in-Chief of the Journal of IAEEE.

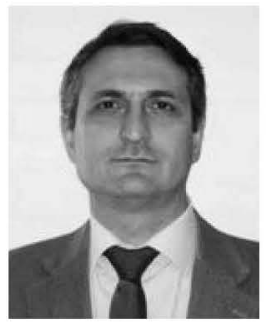

Pedro Rodriguez (S'99-M'04-SM'10-F'13) received the M.Sc. and Ph.D. degrees in electrical engineering from the Technical University of Catalonia (UPC), Barcelona, Spain, in 1994 and 2004, respectively.

He was a Post-Doctoral Researcher with the Center for Power Electronics Systems, Virginia Tech, Blacksburg, VA, USA, in 2005, and the Department of Energy Technology, Aalborg University (AAU), Aalborg, Denmark, in 2006. He joined the faculty at UPC as an Assistant Professor in 1990, where he became the Director of the Department of Electrical Engineering with the Research Center on Renewable Electrical Energy Systems. He was also a Visiting Professor at AAU from 2007 to 2011, acting as a Co-Supervisor of the Vestas Power Program. He still lectures Ph.D. courses at AAU every year. Since 2011, he has been the Head of the Electrical Engineering Division with Abengoa Research, and is still with the UPC as a part-time Professor. He has co-authored one book and more than 100 papers in technical journals and conference proceedings. He is the holder of seven licensed patents. His current research interests include integration of distributed generation systems, smart grids, and design and control of power converters.

Dr. Rodriguez is a member of the Administrate Committee of the IEEE Industrial Electronics Society (IES), the General Chair of the IEEE IES Gold and Student Activities, the Vice Chair of the Sustainability and Renewable Energy Committee of the IEEE Industry Application Society, and a member of the IEEE IES Technical Committee on Renewable Energy Systems. He is an Associate Editor of the IEEE TRANSACTIONS ON POWER ELECTRONICS. 